\title{
Microdissection testicular sperm extraction for men undergoing cancer treatment
}

\section{Jennifer Thomas, Utsav Radia, Jonathan Ramsay \& Channa N Jayasena}

To cite this article: Jennifer Thomas, Utsav Radia, Jonathan Ramsay \& Channa N Jayasena (2016): Microdissection testicular sperm extraction for men undergoing cancer treatment, Expert Review of Quality of Life in Cancer Care

To link to this article: http://dx.doi.org/10.1080/23809000.2016.1181975

Accepted author version posted online: 26

Apr 2016.

Submit your article to this journal $₫$

Q View related articles $\asymp$

View Crossmark data \lceil 
Publisher: Taylor \& Francis \& Copyright ( $)$ Taylor $\&$ Francis

Journal: Expert Review of Quality of Life in Cancer Care

DOI: $10.1080 / 23809000.2016 .1181975$

\section{Review}

Microdissection testicular sperm extraction (mTESE) for men undergoing cancer treatment

Jennifer Thomas, Utsav Radia, Channa N Jayasena

Affiliations: Section of Investigative Medicine, Imperial College London.

Corresponding author for proof and reprints:

Dr. Channa Jayasena

Department of Investigative Medicine,

Imperial College London,

6th Floor, Commonwealth Building,

Hammersmith Hospital,

Du Cane Road,

London W12 ONN,

UK.

Tel: +44 2083833242

Fax: +44 2083833142

c.jayasena@imperial.ac.uk 


\section{Abstract}

Steady improvements in the long term outlook for adolescents and young adults with cancer require a shift in focus towards ensuring quality of life after cancer treatment as well as quantity. An important component of quality of life for many men is the ability to father a biological child. However, direct effects of malignancy, as well as potentially gonadotoxic cancer treatments, render many men azoospermic. Where cryopreservation of a good quality semen sample is not possible or was not offered prior to initiation of treatment, microdissection testicular sperm extraction (mTESE) offers a potential route to biological fatherhood. This review explores current evidence supporting the use of MTESE in patients treated for cancer, as well as some of the barriers and questions that still remain before this technique can form part of routine practice.

\section{Introduction}

The last 40 years have seen steady improvements in the proportion of paediatric and young adult patients surviving long-term after treatment for cancer ${ }^{1,2}$. Increased awareness of signs and symptoms, timely referral with improved access to diagnostic investigations, and advances in treatment with centralisation of services have all contributed to this improved outlook ${ }^{3}$, to the extent that a man diagnosed with testicular cancer in 2011 has a $98 \%$ chance of surviving at least 10 years, compared with just $69.2 \%$ in $1971^{4}$. Whilst there is clearly much to celebrate in these statistics, it is important to recognise the burden on mental and physical health of surviving cancer and its treatment, with a shift in focus towards ensuring quality of life after treatment as well as quantity.

An important component of quality of life for many male cancer survivors is the ability to father children and raise a family. In a large survey of men treated for cancer in the preceding 2 years, $77 \%$ of respondents who were childless at the time of cancer diagnosis expressed a wish to have children in the future ${ }^{5}$. Infertility has an important psychological impact; many men rendered infertile by their cancer treatment experience prolonged grief and distress $^{6}$. Men also worry about how relationships will be affected by disclosing potential infertility ${ }^{7}$. Whilst it is possible to conceive through sperm donation or adoption, most men have a preference to father a biological child ${ }^{8}$. Chemotherapy, radiotherapy and malignancy itself can all adversely affect sperm number and quality, potentially permanently ${ }^{9-11}$, and male survivors of cancer are less than half as likely to father a child as their unaffected siblings ${ }^{12}$. Alkylating agents such as cyclophosphamide, widely used in the treatment of paediatric and adolescent leukaemias, lymphomas and sarcomas are particularly gonadotoxic. A single dosing pulse of cyclophosphamide 
causes long-lasting azoospermia in up to $70 \%$ of male patients ${ }^{13}$. In another study of 67 men treated with chemoand/or radiotherapy, predominantly for lymphomas and testicular cancer, 57\% were rendered permanently azoospermic by their treatment ${ }^{14}$.

In an ideal world, all male patients planning to undergo potentially gonadotoxic cancer treatment would first provide a semen sample for cryopreservation, allowing future use of high quality thawed sperm for intrauterine insemination or in vitro fertilisation. However a number of barriers to this strategy exist. Firstly, despite national guidelines that sperm cryopreservation should be offered to all such men and adolescent boys 15 , a number of studies have shown that the potential effects of cancer treatment on fertility are under-discussed by medical teams and many eligible men are not given this opportunity ${ }^{16,17}$. Low semen quality or azoospermia may already exist due to local, endocrine or autoimmune effects of the cancer itself ${ }^{18}$, and in other cases severe systemic illness induced by malignancy, combined with the urgency of starting treatment, may make producing an ejaculated sample in a hospital setting logistically challenging. Moreover, producing a semen sample is not possible for pre-pubertal boys, who nonetheless stand the same risk as adults of permanent azoospermia following cancer treatment ${ }^{10,19,20}$.

No methods currently exist to restore normal fertility in patients rendered azoospermic by malignancy or following spermatogonial stem cell destruction by cancer therapies. However, histological examination of testicular biopsies suggest that isolated foci of active spermatogenesis may persist despite global testicular failure ${ }^{21}$. Furthermore, assisted reproductive technology (ART) incorporating intracytoplasmic sperm injection (ICSI) can be performed using low numbers of testicular sperm. Therefore, surgical sperm retrieval is implicated as a potential means of allowing men with cancer to have a realistic chance of fathering a biological child. Of these surgical techniques, microdissection testicular sperm extraction (MTESE) balances the greatest likelihood of successful sperm retrieval with an acceptable profile of adverse affects ${ }^{22}$. However, barriers and questions still remain before this specialised technique can form part of routine practice.

\section{Process of mTESE}

During conventional surgical testicular sperm extraction (TESE), multiple samples of testicular tissue are taken through one or more incisions in the tunica albuginea, in the hope that they will contain foci of active 
spermatogenesis ${ }^{23}$. However, this approach runs a significant risk of damage to branches of the testicular artery that run along the anterior surface of the testis to supply the testicular parenchyma ${ }^{24}$. Where prognostic biopsies have shown limited sperm production, large volumes of testicular tissue may be removed to maximise chances of finding viable spermatozoa. This reduction in testicular volume, alongside devascularisation and haematoma formation, can lead to further impairment in testicular function with severely reduced androgen production ${ }^{25} 26$. Moreover in nonobstructive azoospermia, sperm retrieval rate with this method is at best around $40 \%$, with fertilisation rates in subsequent ICSI around $50 \%{ }^{27}$.

mTESE offers several improvements over conventional TESE, increasing sperm yield, whilst minimising adverse effects ${ }^{27,28}$. Based on the finding that seminiferous tubules bearing developing germ cells tend to be larger and more opaque than their sclerotic neighbours that contain sertoli cells only, tissue removal can be targeted to those regions most likely to contain viable sperm. After opening the tunica vaginalis via a transverse midline incision in the scrotum, the tunica albuginea is visualised and reflected. 6-8x magnification allows identification of and avoidance of testicular vessels to preserve blood supply. Direct visualisation of as much as possible of the testicular parenchyma with 20-25x magnification then follows, with excision of small (typically 2-10mg) samples of any large, opaque tubules ${ }^{27,29}$. Variations on this operative technique employ a sagittal incision in the tunica and microdissection between the lobules, sparing coronally orientated vasculature within the testis. Definitive evidence of superiority of either technique is still required. Extracts are stored in optimised sperm transport media and minced with sterile scissors. The resulting sample is passed through a fine gauge angio-catheter to produce a homogeneous suspension for optimal sperm isolation 30 . Ideally aliquots of these samples are examined intra-operatively for the presence of viable sperm by an experienced andrologist. The procedure can be terminated once sufficient spermatozoa are retrieved, otherwise further examination of the same testis may take place, followed if necessary by exploration of the contralateral testis. In one of the largest studies, 1054 men with non-obstructive azoospermia (of varying aetiologies) underwent mTESE, with sperm seen intra-operatively and successfully retrieved in $52.5 \%$. Of the remaining patients in whom the procedure was initially thought to be unsuccessful, more extensive searching in the laboratory with tissue digestion yielded viable spermatozoa in a further 7\%. Using ICSI, there was a subsequent total live birth rate of $26-28 \%{ }^{31}$. 
Practically, mTESE may be performed on a day case or even outpatient basis, and can be performed under local or general anaesthesia ${ }^{27}$. Initial recovery is generally straightforward with a low incidence of early complications such as post-operative bleeding or wound infection ${ }^{23,27}$. In the longer term, several studies have shown that the extensive ultrasonographic abnormalities seen around testicular biopsy sites following conventional TESE are mitigated by a microsurgical approach ${ }^{23,32}$. Nevertheless, in the largest published study addressing endocrine changes in 435 men undergoing mTESE or conventional TESE, a transient and equivalent fall in serum testosterone was seen at 3-6 months following both procedures. On average serum testosterone levels returned to $85 \%$ of initial values within 12 months, but hypogonadism persisted in a minority of patients ${ }^{26}$. This evidence is corroborated by a further, smaller study which suggested that de novo androgen deficiency developed in $16 \%$ of men following mTESE ${ }^{33}$.

\section{Post-chemotherapy azoospermia and mTESE}

Despite the potentially wide application of MTESE for patients with cancer, there exists little published research investigating the outcome of MTESE in azoospermic men following cancer treatment. In the largest case series to date, Schlegel et al ${ }^{22}$ document their experience of MTESE in 73 men with persistent post-chemotherapy azoospermia, with a mean interval post-treatment of 19.9 years. During this study, Sertoli-cell only pattern histology was observed in the majority of patients, which is consistent with previous studies on testicular function following chemotherapy ${ }^{11,19,34}$. However, spermatozoa were successfully retrieved in 27 (37.2\%) patients on initial mTESE, rising to 36 (42.9\%) during repeated procedures; this is surprising when considering that the majority of patients had histological features which failed to detect any active spermatogenesis. In cases with successful sperm retrieval, the reported live birth rate with ICSI was $42 \%$. Thus mTESE plus ICSI allowed 13 men of the $73(17.8 \%)$ included in the study to become biological fathers. These data suggest that MTESE is a potential therapeutic option for patients with azoospermia post-cancer therapy, although further and larger studies are required to define the prognosis for sperm retrieval associated with this approach.

When MTESE has failed to yield viable sperm in patients with post-chemotherapy azoospermia, there is some evidence that medical optimisation of hormonal status may improve likelihood of successful sperm retrieval during a subsequent mTESE procedure. In a small study by Shiraishi et $\mathrm{al}^{35}$, six azoospermic men with failed mTESE following chemotherapy were treated with subcutaneous human chorionic gonadotrophin injections for at least three months 
prior to a repeat mTESE procedure. Increased thickness of the basement membrane and Leydig cell hyperplasia were seen in all patients, with successful sperm retrieval in two. Of note, both of these men had a histological pattern of maturation arrest on initial biopsy; salvage hormonal therapy was not successful in any patients with sertoli-cell only histology. Whilst the very small sample size clearly limits the conclusions that can be drawn from this data, it does reflect findings in a larger sample of patients with other aetiologies of azoospermia suggesting that some testicular histological subtypes at least may be amenable to medical optimization ${ }^{36}$. It is interesting to speculate whether hormonal therapy for azoospermia would potentially improve the chance of sperm retrieval purely in hypogonadal patients, or all azoospermic patients (with or without hypogonadism). Further studies are required to answer this question, which has substantial implications for the optimization of azoospermic cancer survivors prior to mTESE.

Sperm retrieval rate and live pregnancy rate are notably lower overall than in other studies where varying aetiologies of NOA such as Klinefelter syndrome, cryptorchidism and varicocoele, have been included ${ }^{23,27,32,37,38}$. This may be a reflection of the testicular histological patterns of hypospermatogenesis and maturation arrest that are more frequently seen in these groups, and are associated with higher sperm retrieval rates ${ }^{39}$. In keeping with this, all patients with post chemotherapy azoospermia and evidence of hypospermatogenesis on testicular biopsy had successful sperm retrieval. Of note, in this and other studies ${ }^{35}$, sperm retrieval rate was closely associated with cancer type. Despite having just one testicle, $85 \%$ of patients with previous testicular cancer had successful sperm retrieval on initial $\mathrm{mTESE}$, compared to only $14 \%$ of those treated for sarcoma. This is likely to reflect the relatively less gonadotoxic platinum-based treatment regimes used in testicular cancer, with more extensive use of cyclophosphamide and other alkylating agents in sarcomas ${ }^{13,40}$. This may be useful in counselling patients on the likelihood of success before embarking on mTESE. There was no apparent relationship between time since chemotherapy, age, testicular volume or FSH status and likelihood of a positive outcome, although this may relate to the small numbers of patients in the study and would benefit from further research.

Notably absent from these case studies are men who are azoospermic at presentation with cancer, secondary to local, systemic or endocrine effects of malignancy itself. Where testicular function is already compromised prior to the additional insult of potentially gonadotoxic therapies, there is a rational argument for consideration of preemptive mTESE before treatment initiation, to maximise the likelihood of isolating sperm for future use in ART. 
There is currently no published research on the outcomes of MTESE in this group and this should be a focus of ongoing research.

The principle of surgical testicular sperm extraction prior to treatment has already been established in testicular cancer, where testicular tissue excised at orchidectomy can be examined for the presence of viable spermatozoa for use in ART, a procedure known as 'onco-TESE' ${ }^{41}$, 42 . Pitfalls with this technique include the possibility that recovered spermatozoa may have been affected by the biochemical malignant environment, or may come from seminiferous tubules with carcinoma in situ. Moreover as the likelihood of spermatogenesis increases with distance from cancerous tissue, patients in whom the majority of ipsilateral testicular tissue is involved frequently have unsuccessful sperm retrieval $^{43}$. In unilateral disease, concurrent mTESE of the contralateral testis at the time of orchidectomy would supervene these problems, also minimising the risk of hypogonadism that could be anticipated from removal of large volumes of tissue from the contralateral testis as in conventional TESE.

One common concern in young patients treated for cancer is the risk of possible birth defects and genetic abnormalities in their potential offspring ${ }^{7}$ Several large studies have shown that this risk is not higher than in the general population when fertilisation is with ejaculated spermatozoa, whether naturally or through intra-uterine insemination or in vitro fertilisation ${ }^{44-46}$. However high powered evidence that this remains the case for surgically extracted testicular sperm and ICSI is lacking, largely due to the rarity of the procedure in this population. Cytotoxic drugs have been demonstrated to cause extensive genetic and chromosomal abnormalities in ejaculated sperm that can persist up to 18 months after completion of treatment ${ }^{47-49}$, and spermatozoal DNA damage is associated with abnormal blastocyst development in IVF ${ }^{50}$. To minimise these risks, it would be prudent to reserve mTESE for men who remain azoospermic for several years after completion of gonadotoxic therapy, particularly as many men who are initially azoospermic may recover sufficient spermatogenesis for natural conception during this time ${ }^{14}$. 


\section{Expert commentary}

Whilst mTESE offers the best available chance for men who are azoospermic following cancer treatment to father a biological child, it is important to remember that the ultimate likelihood of success (ie. live birth) is consistently below 1 in $5^{22,35}$. In contrast, where men have had the opportunity to bank sperm prior to initiation of treatment, close to $80 \%$ of those who subsequently use their samples in ART will achieve paternity ${ }^{51}$, highlighting the importance of offering sperm cryopreservation where practicable. Given that fertility outcomes are not affected by duration of storage of cryopreserved sperm ${ }^{52} 53$, perceived awkwardness or lack of immediate relevance should not prevent discussion of fertility with boys who are adolescent at time of diagnosis; these patients make up a significant proportion of those subsequently referred for MTESE and frequently report a lack of awareness of the impact of treatment on their fertility potential ${ }^{54,55}$. In pre-pubescent boys where sperm cryopreservation is impossible, consideration should be given to pre-emptive testicular tissue harvesting for potential future use in experimental treatments such as fspermatogonial stem cell (SSC) banking. Surgical recovery of pre-pubertal testicular tissue may be combined with other procedures requiring general anaesthesia such as venous catheter insertion or bone marrow sampling, minimising trauma and anaesthetic risk. ${ }^{56}$ Whilst realisation of the fertility potential of SCCS remains as yet undemonstrated in humans, significant progress has been made in animal studies, with demonstration of restoration of functional spermatogenesis following autotransplantation of a suspension of frozenthawed SCCs into the testes of rhesus monkeys rendered azoospermic with alkylating agents ${ }^{57}$. Such strategies may well offer an alternative to mTESE when these and future generations of young cancer patients reach reproductive age.

In counselling men and their partners on the likelihood of success with mTESE, it is worth considering several factors that may make actual paternity rates somewhat lower than the best reported in published studies. Firstly, there is evidence of a substantial learning curve in surgical experience, with the best sperm retrieval rates seen after a surgeon has performed at least 50 such operations ${ }^{58}$. Where availability and uptake of the procedure is low, the best outcomes may be seen if cases are channelled to large centres with higher throughput.

In the largest case series of mTESE in post-chemotherapy azoospermia, ICSI was performed using fresh sperm extracted on the day of or day before egg harvesting; requiring synchronisation with egg harvesting in female partners ${ }^{22}$. This is unlikely to be feasible in most healthcare settings, and indeed the current standard in the UK is to use cryopreserved harvested sperm for ICSI. The impact of this difference on overall success rates is yet to be seen. 
However unlike with ejaculated sperm where there is clear evidence for a detrimental effect of freeze-thaw cycles ${ }^{59}$, the use of ICSI rather than IVF may supervene some of these problems, with similar fertilisation rates for fresh and cryopreserved sperm ${ }^{60,61}$.

The psychological impact of mTESE should also be carefully considered before offering it to men rendered azoospermic following cancer therapy. Surgical sperm retrieval is an emotionally demanding procedure which is deemed successful if sperm are collected. However, patients with successful sperm retrieval do not always have successful assisted reproductive technologies (ART), and successful ART does not always lead to a live birth. It is therefore important to counsel men undergoing mTESE for cancer to be fully aware of numerous steps which must be negotiated to allow conception. Nevertheless, for many men the chance to father a biological child is of central importance to their wellbeing and psychological recovery after cancer ${ }^{8}$. Offering as it does a realistic chance of achieving this goal in a group of men traditionally seen as infertile, wider application of mTESE should be considered as we move towards a greater appreciation of the importance of quality of life after cancer treatment. 


\section{Five year view}

As expectations of cancer survival increase, patients will increasingly demand long-term quality of life to be central to their management plan. We anticipate that MTESE will become increasingly available to the minority of male cancer survivors wishing to father a child, who are azoospermic and have no cryopreserved sperm. Larger, multicentre studies are likely to emerge, giving clinicians and patients better information about the potential benefits and risks (principally the risk of failed sperm retrieval) associated with mTESE. Although currently lacking, data on the effectiveness of pre-cancer therapy sperm retrieval may become available. Finally, greater expert consensus and clinical guidance is required to standardise the quality of fertility treatment including $\mathrm{mTESE}$, in male survivors of cancer.

\section{Key Issues}

- The future ability to father a child is of central importance to many men, but cancer and its treatment render a significant proportion of male cancer patients azoospermic.

- Testes of azoospermic men may contain foci of active spermatogenesis. Where cryopreservation of a good quality semen sample was not possible or was not offered prior to initiation of treatment, testicular sperm extraction combined with ICSI may allow azoospermic men to achieve biological fatherhood.

- Microdissection testicular sperm extraction offers advantages over conventional TESE. Identification and isolation of seminiferous tubules bearing developing germ cells increases likelihood of successful sperm retrieval, whilst minimising the volume of testicular tissue removed and the risk of damage to testicular vasculature.

- Sperm retrieval rate and live birth rate following mTESE for men who are azoospermic after cancer treatment appear lower than in azoospermia of other aetiologies. Success rate varies between cancer type and treatment modalities, with cyclophosphamide-based chemotherapy regimens being particularly associated with unsuccessful sperm retrieval. Medical optimisation of hormonal status may improve outcomes, but requires further research.

- Further research is required into the potential role of $\mathrm{mTESE}$ prior to initiation of therapy in men who are azoospermic at the time of cancer diagnosis. 
- Whilst mTESE offers the best available chance for men who are azoospermic following cancer treatment to father a biological child, the ultimate likelihood of success (ie. live birth) remains relatively low (less than 1 in 5). Where possible, sperm cryopreservation before treatment remains the gold standard.

- $\quad$ The psychological impact of mTESE should also be carefully considered before offering it to men rendered azoospermic following cancer therapy. It is important to counsel men undergoing mTESE for cancer to be fully aware of numerous steps which must be negotiated to allow conception.

\section{Financial and competing interests disclosure}

The Section of Endocrinology and Investigative Medicine is funded by grants from the MRC, BBSRC, NIHR, an Integrative Mammalian Biology (IMB) Capacity Building Award, an FP7- HEALTH- 2009-241592 EuroCHIP grant and is supported by the NIHR Biomedical Research Centre Funding Scheme. The views expressed are those of the author(s) and not necessarily those of the NHS, the NIHR or the Department of Health. JT is funded by The Northwest Thames Academic Foundation Programme. The authors have no other relevant affiliations or financial involvement with any organization or entity with a financial interest in or financial conflict with the subject matter or materials discussed in the manuscript apart from those disclosed. 
La Vecchia, C. et al. Cancer mortality in Europe, 2000-2004, and an overview of trends since 1975. Annals of oncology : official journal of the European Society for Medical Oncology / ESMO 21, 1323-1360, doi:10.1093/annonc/mdp530 (2010).

Stiller, C. A. et al. Geographical patterns of childhood cancer incidence in Europe, 1988-1997. Report from the Automated Childhood Cancer Information System project. Eur J Cancer 42, 1952-1960, doi:10.1016/j.ejca.2006.05.017 (2006).

Walters, S. et al. Is England closing the international gap in cancer survival[quest]. Br J Cancer 113, 848-860, doi:10.1038/bjc.2015.265 (2015).

Quaresma, M., Coleman, M. P. \& Rachet, B. 40-year trends in an index of survival for all cancers combined and survival adjusted for age and sex for each cancer in England and Wales, 1971-2011: a population-based study. The Lancet 385, 1206-1218, doi:http://dx.doi.org/10.1016/S0140-6736(14)61396-9.

Schover, L. R., Brey, K., Lichtin, A., Lipshultz, L. I. \& Jeha, S. Knowledge and experience regarding cancer, infertility, and sperm banking in younger male survivors. Journal of clinical oncology: official journal of the American Society of Clinical Oncology 20, 1880-1889 (2002).

Rieker, P. P., Fitzgerald, E. M. \& Kalish, L. A. Adaptive behavioral responses to potential infertility among survivors of testis cancer. Journal of clinical oncology : official journal of the American Society of Clinical Oncology 8, 347-355 (1990).

Nilsson, J. et al. 'Will I be able to have a baby?' Results from online focus group discussions with childhood cancer survivors in Sweden. Human reproduction (Oxford, England) 29, 2704-2711, doi:10.1093/humrep/deu280 (2014).

Tschudin, S. \& Bitzer, J. Psychological aspects of fertility preservation in men and women affected by cancer and other life-threatening diseases. Human reproduction update 15, 587-597, doi:10.1093/humupd/dmp015 (2009).

DeSantis, M., Albrecht, W., Holtl, W. \& Pont, J. Impact of cytotoxic treatment on long-term fertility in patients with germ-cell cancer. International journal of cancer. Journal international du cancer 83, 864-865 (1999).

Mackie, E. J., Radford, M. \& Shalet, S. M. Gonadal function following chemotherapy for childhood Hodgkin's disease. Medical and pediatric oncology 27, 74-78, doi:10.1002/(sici)1096-911x(199608)27:2<74::aidmpo2>3.0.co;2-q (1996).

11 Rowley, M. J., Leach, D. R., Warner, G. A. \& Heller, C. G. Effect of graded doses of ionizing radiation on the human testis. Radiation research 59, 665-678 (1974).

12 Green, D. M. et al. Fertility of male survivors of childhood cancer: a report from the Childhood Cancer Survivor Study. Journal of clinical oncology : official journal of the American Society of Clinical Oncology 28, 332-339, doi:10.1200/jco.2009.24.9037 (2010). Kenney, L. B., Laufer, M. R., Grant, F. D., Grier, H. \& Diller, L. High risk of infertility and long term gonadal damage in males treated with high dose cyclophosphamide for sarcoma during childhood. Cancer 91, 613621 (2001).

14 Schmidt, K. L. et al. Assisted reproduction in male cancer survivors: fertility treatment and outcome in 67 couples. Human reproduction (Oxford, England) 19, 2806-2810, doi:10.1093/humrep/deh518 (2004). Excellence, N. I. f. H. a. C. (2013).

Quinn, G. P. et al. Physician referral for fertility preservation in oncology patients: a national study of practice behaviors. Journal of clinical oncology : official journal of the American Society of Clinical Oncology 27, 5952-5957, doi:10.1200/jco.2009.23.0250 (2009).

17 Schover, L. R., Brey, K., Lichtin, A., Lipshultz, L. I. \& Jeha, S. Oncologists' attitudes and practices regarding banking sperm before cancer treatment. Journal of clinical oncology : official journal of the American Society of Clinical Oncology 20, 1890-1897 (2002).

18 Agarwal, A. \& Allamaneni, S. S. Disruption of spermatogenesis by the cancer disease process. Journal of the National Cancer Institute. Monographs, 9-12, doi:10.1093/jncimonographs/lgi005 (2005). Aubier, F. et al. Male gonadal function after chemotherapy for solid tumors in childhood. Journal of clinical oncology : official journal of the American Society of Clinical Oncology 7, 304-309 (1989). Thomson, A. B. et al. Semen quality and spermatozoal DNA integrity in survivors of childhood cancer: a casecontrol study. Lancet (London, England) 360, 361-367 (2002). 
Jow, W. W., Steckel, J., Schlegel, P. N., Magid, M. S. \& Goldstein, M. Motile sperm in human testis biopsy specimens. Journal of andrology 14, 194-198 (1993).

Hsiao, W. et al. Successful treatment of postchemotherapy azoospermia with microsurgical testicular sperm extraction: the Weill Cornell experience. Journal of clinical oncology : official journal of the American Society of Clinical Oncology 29, 1607-1611, doi:10.1200/jco.2010.33.7808 (2011). ** of considerable interest**

The largest study thus far into mTESE in men who are azoospermic after cancer treatment.

Okada, H. et al. Conventional versus microdissection testicular sperm extraction for nonobstructive azoospermia. The Journal of urology 168, 1063-1067, doi:10.1097/01.ju.0000025397.03586.c4 (2002). * of interest * Thorough comparison of the two techniques

Jarow, J. P. Clinical significance of intratesticular arterial anatomy. Journal of Urology 145, 777-779 (1991). Schlegel, P. N. \& Su, L. M. Physiological consequences of testicular sperm extraction. Human Reproduction 12, 1688-1692, doi:10.1093/humrep/12.8.1688 (1997).

Ramasamy, R., Yagan, N. \& Schlegel, P. N. Structural and functional changes to the testis after conventional versus microdissection testicular sperm extraction. Urology 65, 1190-1194, doi:10.1016/j.urology.2004.12.059 (2005).

Schlegel, P. N. Testicular sperm extraction: microdissection improves sperm yield with minimal tissue excision. Human reproduction (Oxford, England) 14, 131-135 (1999).

Bernie, A. M., Mata, D. A., Ramasamy, R. \& Schlegel, P. N. Comparison of microdissection testicular sperm extraction, conventional testicular sperm extraction, and testicular sperm aspiration for nonobstructive azoospermia: a systematic review and meta-analysis. Fertility and sterility 104, 1099-1103.e1093, doi:http://dx.doi.org/10.1016/i.fertnstert.2015.07.1136 (2015).

Ishikawa, T. Surgical recovery of sperm in non-obstructive azoospermia. Asian Journal of Andrology 14, 109115, doi:10.1038/aja.2011.61 (2012).

Ostad, M., Liotta, D., Ye, Z. \& Schlegel, P. N. Testicular sperm extraction for nonobstructive azoospermia: results of a multibiopsy approach with optimized tissue dispersion. Urology 52, 692-696 (1998).

Ramasamy, R. et al. Role of tissue digestion and extensive sperm search after microdissection testicular sperm extraction. Fertility and sterility 96, 299-302, doi:http://dx.doi.org/10.1016/i.fertnstert.2011.05.033 (2011).

Amer, M., Ateyah, A., Hany, R. \& Zohdy, W. Prospective comparative study between microsurgical and conventional testicular sperm extraction in non-obstructive azoospermia: follow-up by serial ultrasound examinations. Human reproduction (Oxford, England) 15, 653-656 (2000).

Everaert, K. et al. Long term effects of micro-surgical testicular sperm extraction on androgen status in patients with non obstructive azoospermia. BMC Urology 6, 9-9, doi:10.1186/1471-2490-6-9 (2006).

Berthelsen, J. G. \& Skakkebaek, N. E. Gonadal function in men with testis cancer. Fertility and sterility 39, 6875 (1983).

35 Shiraishi, K. \& Matsuyama, H. Microdissection testicular sperm extraction and salvage hormonal treatment in patients with postchemotherapy azoospermia. Urology 83, 100-106, doi:10.1016/j.urology.2013.08.043 (2014).

36 Shiraishi, K., Ohmi, C., Shimabukuro, T. \& Matsuyama, H. Human chorionic gonadotrophin treatment prior to microdissection testicular sperm extraction in non-obstructive azoospermia. Human reproduction (Oxford, England) 27, 331-339, doi:10.1093/humrep/der404 (2012).

Tsujimura, A. et al. Conventional multiple or microdissection testicular sperm extraction: a comparative study. Human reproduction (Oxford, England) 17, 2924-2929 (2002).

38 Ramasamy, R. et al. High serum FSH levels in men with nonobstructive azoospermia does not affect success of microdissection testicular sperm extraction. Fertility and sterility 92, 590-593,

doi:10.1016/j.fertnstert.2008.07.1703 (2009).

Glina, S. \& Vieira, M. Prognostic factors for sperm retrieval in non-obstructive azoospermia. Clinics 68, 121124, doi:10.6061/clinics/2013(Sup01)13 (2013).

Meistrich, M. L., Wilson, G., Brown, B. W., da Cunha, M. F. \& Lipshultz, L. I. Impact of cyclophosphamide on long-term reduction in sperm count in men treated with combination chemotherapy for Ewing and soft tissue sarcomas. Cancer 70, 2703-2712 (1992).

Schrader, M. et al. "Onco-tese": testicular sperm extraction in azoospermic cancer patients before chemotherapy-new guidelines? Urology 61, 421-425 (2003). * Of interest *

Descombe, L. et al. Testicular sperm extraction in a single cancerous testicle in patients with azoospermia: a case report. Fertility and sterility 90, 443.e441-444, doi:10.1016/j.fertnstert.2007.07.1308 (2008). 
Suzuki, K., Shin, T., Shimomura, Y., Iwahata, T. \& Okada, H. Spermatogenesis in tumor-bearing testes in germ cell testicular cancer patients. Human reproduction (Oxford, England) 30, 2853-2858, doi:10.1093/humrep/dev250 (2015). offspring of survivors of childhood cancer. Archives of pediatrics \& adolescent medicine 151, 379-383 (1997). Mulvihill, J. J. et al. Cancer in offspring of long-term survivors of childhood and adolescent cancer. Lancet (London, England) 2, 813-817 (1987).

Senturia, Y. D. \& Peckham, C. S. Children fathered by men treated with chemotherapy for testicular cancer. Eur J Cancer 26, 429-432 (1990).

47 Chatterjee, R., Haines, G. A., Perera, D. M., Goldstone, A. \& Morris, I. D. Testicular and sperm DNA damage after treatment with fludarabine for chronic lymphocytic leukaemia. Human reproduction (Oxford, England) 15, 762-766 (2000).

De Mas, P. et al. Increased aneuploidy in spermatozoa from testicular tumour patients after chemotherapy with cisplatin, etoposide and bleomycin. Human reproduction (Oxford, England) 16, 1204-1208 (2001). Frias, S. et al. NOVP chemotherapy for Hodgkin's disease transiently induces sperm aneuploidies associated with the major clinical aneuploidy syndromes involving chromosomes $X, Y, 18$, and 21. Cancer research 63, 44-51 (2003).

Seli, E., Gardner, D. K., Schoolcraft, W. B., Moffatt, O. \& Sakkas, D. Extent of nuclear DNA damage in ejaculated spermatozoa impacts on blastocyst development after in vitro fertilization. Fertility and sterility 82, 378-383, doi:10.1016/j.fertnstert.2003.12.039 (2004). Muller, I., Oude Ophuis, R. J. A., Broekmans, F. J. M. \& Lock, T. M. T. W. Semen cryopreservation and usage rate for assisted reproductive technology in 898 men with cancer. Reproductive BioMedicine Online 32, 147153, doi:http://dx.doi.org/10.1016/i.rbmo.2015.11.005 (2016).

Clarke, G. N., Liu de, Y. \& Baker, H. W. Recovery of human sperm motility and ability to interact with the human zona pellucida after more than 28 years of storage in liquid nitrogen. Fertility and sterility $\mathbf{8 6}, \mathbf{7 2 1 -}$ 722, doi:10.1016/j.fertnstert.2006.01.050 (2006). Horne, G. et al. Live birth with sperm cryopreserved for 21 years prior to cancer treatment: case report. Human reproduction (Oxford, England) 19, 1448-1449, doi:10.1093/humrep/deh249 (2004). de Vries, M. C., Bresters, D., Engberts, D. P. Wit, J. M. \& van Leeuwen, E. Attitudes of physicians and parents towards discussing infertility risks and semen cryopreservation with male adolescents diagnosed with cancer. Pediatric blood \& cancer 53, 386-391, doi:10.1002/pbc.22091 (2009). Neal, M. S. et al. Effectiveness of sperm banking in adolescents and young adults with cancer: a regional experience. Cancer 110, 1125-1129, doi:10.1002/cncr.22889 (2007).

56 Picton, H. M. et al. A European perspective on testicular tissue cryopreservation for fertility preservation in prepubertal and adolescent boys. Human reproduction (Oxford, England) 30, 2463-2475, doi:10.1093/humrep/dev190 (2015). * of interest* Up to date and thorough review of the current status of SSC transplantation and associated fertility preservation strategies.

57 Hermann, B. P. et al. Spermatogonial stem cell transplantation into rhesus testes regenerates spermatogenesis producing functional sperm. Cell stem cell 11, 715-726, doi:10.1016/j.stem.2012.07.017 (2012).

58 Ishikawa, T., Nose, R., Yamaguchi, K., Chiba, K. \& Fujisawa, M. Learning curves of microdissection testicular sperm extraction for nonobstructive azoospermia. Fertility and sterility 94, 1008-1011, doi:10.1016/j.fertnstert.2009.03.108 (2010). O'Connell, M., McClure, N. \& Lewis, S. E. The effects of cryopreservation on sperm morphology, motility and mitochondrial function. Human reproduction (Oxford, England) 17, 704-709 (2002).

60 Kalsi, J. et al. Analysis of the outcome of intracytoplasmic sperm injection using fresh or frozen sperm. BJU international 107, 1124-1128, doi:10.1111/j.1464-410X.2010.09545.x (2011).

61 Ulug, U., Bener, F., Karagenc, L., Ciray, N. \& Bahceci, M. Outcomes in couples undergoing ICSI: comparison between fresh and frozen-thawed surgically retrieved spermatozoa. International journal of andrology 28, 343-349, doi:10.1111/j.1365-2605.2005.00559.x (2005). 
Table 1 - Incidence of azoospermia and sperm retrieval rate by cancer type and typical treatment regime

\begin{tabular}{|c|c|c|c|}
\hline Type of Cancer & $\begin{array}{l}\text { Typical Regimens }\{\text { Howell, } \\
2001 \# 78\}\end{array}$ & Proportion with azoospermia & $\begin{array}{l}\text { Sperm Retrieval rate in } \\
\text { mTESE }\{\text { Hsiao, } 2011 \# 31\}\end{array}$ \\
\hline Hodgkin's Lymphoma & $\begin{array}{l}\text { M VPP } \\
\text { MOPP } \\
\text { COPP } \\
\text { ABVD }\end{array}$ & $\begin{array}{l}53 \%(n=19)\{\text { Romerius, } 2011 \\
\# 79\}\end{array}$ & $26 \%(n=27)$ \\
\hline Non-Hodgkins Lymphoma & $\begin{array}{l}\text { CHOP } \\
\text { VAPEC-B } \\
\text { VACOP-B } \\
\text { MACOP-B } \\
\text { VEEP }\end{array}$ & $\begin{array}{l}11 \%(\mathrm{n}=21)\{\text { Romerius, } 2011 \\
\# 79\}\end{array}$ & $36 \%(n=11)$ \\
\hline Testicular Cancer & $\begin{array}{l}\text { Cisplatin/ } \\
\text { Carboplatin }\end{array}$ & $\begin{array}{l}11 \%(n=21)\{\text { Romerius, } 2011 \\
\# 79\}\end{array}$ & $86 \%(n=7)$ \\
\hline Sarcoma & $\begin{array}{l}\text { VAC } \\
\text { Adria-VAC }\end{array}$ & $\begin{array}{l}59 \%(n=17)\{\text { K enney, } 2001 \\
\# 55\}\end{array}$ & $14 \%(n=7)$ \\
\hline
\end{tabular}

$M V P P=$ mustine, vinblastine, procarbazine and prednisolone; $M O P P=$ mustine, vincristine, procarbazine and prednisolone; $C O P P=$ cyclophosphamide, vincristine, procarbazine and prednisolone; $\mathrm{ABVD}=\mathrm{A}$ driamycin, bleomycin, vinblastine and dacarbazine; $\mathrm{CHOP}=$ cyclophosphamide, doxorubicin, vincristine and prednisolone; VA PEC-B = vincristine, doxorubicin, prednisolone, etoposide, cyclophosphamide and bleomycin, VACOP-B = vinblastine, doxorubicin, prednisolone, vincristine, cyclophosphamide and bleomycin, $M A C O P-B=$ mustine in place of vinblastine, $V E E P=$ vincristine, etoposide, epirubicin and prednisolone, $V A C=$ vincristine, actinomycin-D, cyclophosphamide; A dria-V AC = addition of adriamycin. 